\title{
Deguelin, a novel anti-tumorigenic agent targeting apoptosis, cell cycle arrest and anti-angiogenesis for cancer chemoprevention (Review)
}

\author{
YING WANG, WENLI MA and WENLING ZHENG \\ Institute of Genetic Engineering, Southern Medical University, Tonghe, Guangzhou 510515, P.R. China
}

Received May 23, 2012; Accepted July 24, 2012

DOI: $10.3892 / \mathrm{mco} .2012 .36$

\begin{abstract}
Deguelin is a natural compound of the flavonoid family products isolated from Derris trifoliata Lour. or Mundulea sericea (Leguminosae). It exhibited significant antitumorigenesis and anti-proliferative activity in various types of cancer both in vitro and in vivo. Deguelin induced cell apoptosis by blocking anti-apoptotic pathways, such as PI3K-Akt, IKK-I $\kappa \mathrm{B} \alpha-\mathrm{NF}-\kappa \mathrm{B}$ and AMPK-mTOR-survivin, while inhibiting tumor cell propagation and malignant transformation through p27-cyclinE-pRb-E2F1 cell cycle control and HIF-1 $\alpha$ VEGF anti-angiogenic pathways. In pre-clinical trials, deguelin markedly decreased the tumor incidence. These biological findings identified deguelin as a novel anti-tumorigenic agent targeting apoptosis, cell cycle arrest and anti-angiogenesis for cancer chemoprevention and chemotherapy.
\end{abstract}

\section{Contents}

1. Introduction

2. Deguelin induced tumor cell death by targeting apoptosis

3. Deguelin inhibited tumor cell growth by targeting cell cycle arrest

4. Deguelin induced tumor cell death by targeting anti-angiogenesis

5. Deguelin was applied in pre-clinical and clinical trials

6. Conclusion

\section{Introduction}

Growing attention has been focused on the application of natural flavonoids in the chemoprevention and chemotherapy

Correspondence to: Professor Wenli Ma or Professor Wenling Zheng, Institute of Genetic Engineering, Southern Medical University, Tonghe, Guangzhou 510515, P.R. China

E-mail:wenli668@gmail.com

E-mail: gendustry@gmail.com

Key words: deguelin, anti-tumorigenesis, apoptosis, cell cycle arrest, anti-angiogenesis of malignant diseases. Several plant rotenoid compounds, such as rotenone, deguelin and tephrosin, have previously been reported to be effective against tumorigenesis, metastasis and invasion (1-3). Deguelin [(7aS, BaS)-13, 13a-dihydro-9, 10-dimethoxy-3, 3-dimethyl-3H-bis [1] benzo-prano [3, 4-b: 6', 5'-e] pyran-7 (7aH)-one] is a natural rotenoid extracted from several plants, including Derris trifoliata Lour.(Leguminosae), Mundulea sericea (Leguminosae) and Tephrosia vogelii Hook.f. (Leguminosae) (Fig. 1). In pre-clinical and clinical trials, deguelin has shown effective anti-tumorigenic and anti-angiogenic activities in various types of cancers.

Rotenone is a highly-toxic deguelin-analogue, utilized as an insecticide and inducer of Parkinson's disease (4). As the old inhibitor of mitochondrial complex I(NADH), rotenone contributes to electron transport chain-blocking and ATP depletion, as well as cell toxicity. Despite their similar structure, the differences in the way deguelin and rotenone function are notable. Numerous studies confirmed that deguelin activated the virus signaling pathway for inhibiting tumor cell proliferation with no or less toxicity (5-8), while demonstrating the highest activity in inhibiting DMBA-induced tumorigenesis compared with

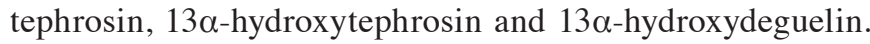
No sign of overt toxicity has been observed at the dose of 2-4 $\mathrm{mg} / \mathrm{kg}(5)$.

Deguelin is a well-known PI3K/Akt inhibitor, capable of specifically binding to the ATP pocket of heat-shock protein $90 \alpha(\mathrm{Hsp} 90 \alpha)$, thus suppressing the function of a variety of Hsp90 client proteins. In the comprehensive signaling network of tumorigenesis, metastasis, apoptosis and angiogenesis, deguelin is pivotal in inducing apoptotic cell death and prohibiting their malignant transformation. Deguelin has been reported to kill cancer cells or inhibit tumor growth through targeting apoptosis, cell cycle arrest and anti-angiogenesis, including PI3K-Akt, IKK-I $\kappa \mathrm{B} \alpha-\mathrm{NF}-\kappa \mathrm{B}$ and AMPK-mTOR-survivin pathways. These findings suggest deguelin to be a novel anti-tumorigenic agent for cancer therapeutic intervention.

\section{Deguelin induced tumor cell death by targeting apoptosis}

Targeting of the PI3K-Akt anti-apoptotic signaling pathway. Apoptosis is a crucial step in cancer therapy and prevention, whereby cells undergo programmed death to eliminate the 
damaged and harmful cells and restrict cell proliferation. Since dysregulated apoptosis is considered to be necessary for tumor development, apoptosis, a pivotal mechanism for normal homeostasis, is crucial in the therapy of various tumors. Therefore, key regulatory components involved in apoptotic or anti-apoptotic pathways are necessary with a view to developing new targeting agents, with fewer side effects, eliminating cancer cells (9).

Deguelin is known to induce apoptosis by disrupting antiapoptosis signaling, such as the PI3K/Akt pathway in lung and breast cancer. The PI3K/Akt pathway and its downstream targets are both important for cell survival and proliferation. In 2003, Chun et al (10) reported that subsequent to deguelin treatment $\left(10^{-7} \mathrm{M}\right)$ premalignant and malignant human bronchial epithelial (HBE) cells showed morphologic changes typical of apoptosis, in contrast to healthy HBE cells. Furthermore, deguelin was confirmed to have induced apoptosis by inhibiting PI3K activity and downregulating the pAkt-level, with no effects on MAPK pathways (10). Subsequent studies have demonstrated that deguelin induced apoptosis by inhibiting the PI3K/Akt pathway in human leukaemia HL-60, U937 and human breast cancer MCF-7 cells (11-13).

In the PI3K-Akt pathway, deguelin treatment resulted in a reduced pAkt level, inhibiting the activity of the pro-apoptotic proteins Bad and Bax by phosphorylation. Bad and Bax are crucial for activating caspase-3, as well as apoptotic downstream targets. Deguelin treatment offsets pAkt inhibition of $\mathrm{Bad}$ and Bax, and the caspase cascade of apoptosis is initiated $(10,14)$.

Targeting of the IKK-I $\mathrm{K} B \alpha-N F-\kappa B$ anti-apoptotic signaling pathway. Nuclear factor- $\kappa \mathrm{B}$ (NF- $\kappa \mathrm{B})$ is a family of well-known transcription factors involved in key signaling pathways, such as acquired immunity, inflammation, stress and carcinogenesis. One pivotal function of NF- $\mathrm{kB}$ is its ability to block cell apoptosis (15). Several studies showed that in deguelin-induced apoptosis, NF-кB activation was suppressed (16-20). In 2006, Nair et al (16) demonstrated that deguelin inhibited IKK activation to protect $I \kappa B \alpha$ from degradation in a variety of cell types. IKK was necessary for I $\mathrm{I} B \alpha$ phosphorylation as well

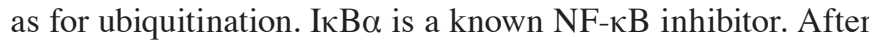
deguelin-incubation, increasingly active I $\mathrm{B} \alpha$ masked the nuclear localization signals of NF- $\mathrm{BB}$. ІкB $\alpha$ was kept outside the nucleus and deprived of the ability to bind to DNA (16). Further investigation suggested that deguelin also suppressed the expression of the downstream targets of NF- $\mathrm{KB}$, including numerous anti-apoptotic proteins, such as Bcl-2, Bcl-xl, Bfl-1, IAP1/2 and TRAF-1 (17).

Nair et al (16) also suggested that the upstream molecules of NF- $\kappa \mathrm{B}$ might be modulated by deguelin. Those authors transiently transfected NF- $\mathrm{\kappa B}$-regulated reporter plasmid, TNFR1, TRAF2, TRADD, NIK and p65 expression plasmids into cancer cells and examined NF- $\mathrm{kB}$-dependent SEAP expression after deguelin treatment. Deguelin was found to have blocked NF- $\kappa$ B activation induced by TNFR1, TRAF2, TRADD and NIK, although not by p65, compared to the control group. The results demonstrated that deguelin acted upstream of p65 in the NF-kB signaling pathway (16).

In contrast to Nair et al (16), Geeraerts et al (18) demonstrated that deguelin promoted I $\kappa \mathrm{B} \alpha$ degradation in B-chronic lympho-

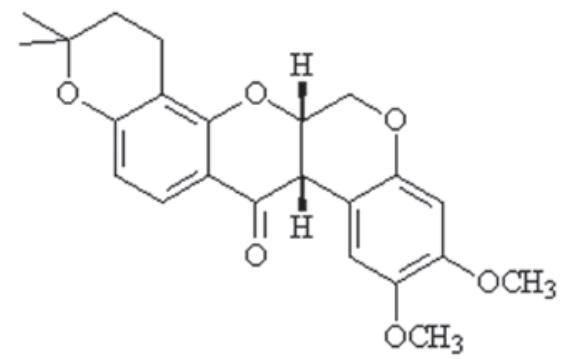

Figure 1. The structure of deguelin.

cytic leukemia (B-CLL) cells. Nevertheless, Chen et al (19) demonstrated that deguelin inhibited the I $\kappa \mathrm{B} \alpha$ expression and induced apoptosis by interacting with the NF- $\kappa \mathrm{B}$ pathway in leukemia cells. Despite these results, the precise mechanism underlying the I $\kappa \mathrm{B} \alpha$ downregulation and the subsequent NF- $\kappa \mathrm{B}-$ inactivation-related apoptosis has yet to be determined. The studies conducted, however, confirmed the efficacy of deguelin on apoptotic cell death through the NF- $\mathrm{kB}$ signaling pathway.

Targeting of the AMPK-mTOR-survivin anti-apoptotic signaling pathway. Apart from the PI3K-Akt and IKK-ІкB $\alpha-$ NF- $\mathrm{KB}$ pathways, deguelin also blocked cell survival through AMPK/mTOR/survivin signaling in HBE cells (21). In this pathway, the first target of deguelin was the AMP-activated protein kinase (AMPK), an enzyme for maintaining cellular energy homeostasis. AMPK activation is correlated with the increase of the ADP/ATP ratio. Various studies have confirmed that sustained activation of AMPK inhibited cell proliferation and induced apoptosis (22-24), since cell growth and mitosis are major consumers of ATP. Recent studies have also demonstrated that activated AMPK-mediated chemotherapy-induced cell apoptosis in bladder cancer or melanoma cells $(25,26)$. These data shed light on the precise mechanism of deguelin induced apoptosis. In HBE cells, deguelin activated AMPK by ATP depletion and inhibited Akt activation. Conversely, the suppressed Akt promoted the function of AMPK, causing the activation of tuberous sclerosis complex 2 (TSC2), a major tumor suppressor, which in turn inhibited mTOR and its upstream protein, p70S6, and activated 4E-BP1. The latter two events suppressed survivin expression. Survivin is an another important molecule regulating cell division and protecting cells from normal death. Survivin plays a key role in tumor immune escape and anti-apoptosis. Taken together, deguelin inhibits mTOR-mediated survivin expression. The ultimate chemopreventive effect of deguelin is owed to its blocking malignant transformation and inducing apoptosis in lung cancer cells.

\section{Deguelin inhibited tumor cell growth by targeting cell cycle arrest}

Cell cycle regulator abnormalities are important characteristics of the progression of human cancers. The loss of control over cell cycle checkpoints contributed significantly to cell proliferation. These checkpoints maintain gene expression in a coordinated manner. Thus, several studies conducted on chemoprevention agents focus on rescuing the cell cycle 


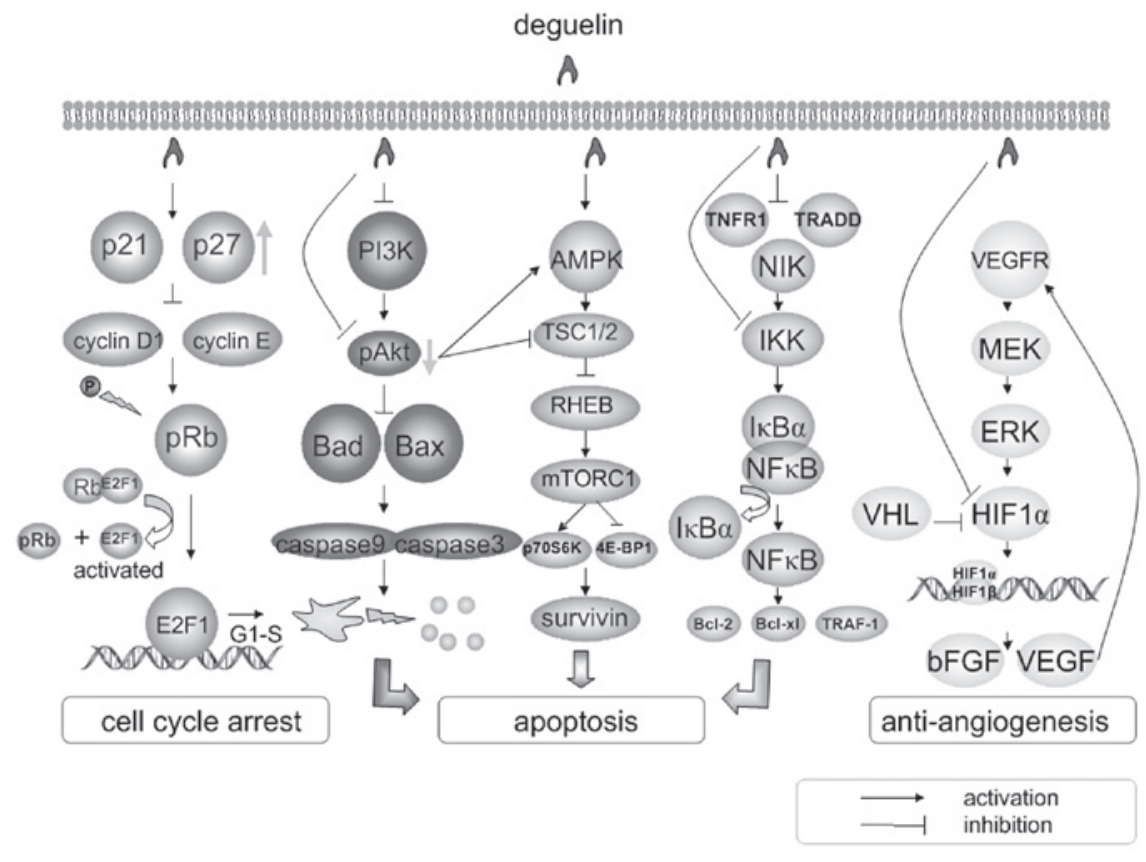

Figure 2. Schematic model of deguelin, a novel anti-tumorigenic agent targeting cell cycle arrest, apoptosis and anti-angiogeneric pathways.

regulators, by targeting the p27-cyclin E-pRb-E2F1 cell cycle control signaling pathway.

Deguelin has been found to regulate cell cycle in colon cancer cells by stimulating p27 expression. p27 is a cyclindependent kinase (CDK) inhibitor, identified as a crucial factor in the cell cycle regulatory cascade, and found to be involved in the prognosis of several types of cancer. Western blot analysis demonstrated that deguelin mediated p27 overexpression resulting in cyclin E inactivation and consequently, pRb dephosphorylation. pRb acts as a tumor suppressor, while hypophosphorylated $\mathrm{pRb}$ is able to bind to E2F1 and inhibit its action. Therefore, deguelin increased the p27 expression and arrested the E2F1 release, contributing to a G1-S phase cell cycle arrest at the end (27). In pre-malignant HBE cells, deguelin treatment also increased the expression of p21 and p27, in a dose-dependent manner (28). According to Murillo et al (29) and Liu et al (30), deguelin induced cyclinD1/pRb decrease as well as subsequent G1-S phase arrest in breast cancer MDA-MB-231 and lymphoma Daudi cells. These data support the cell cycle blocking effects of deguelin on cancer cells. Nevertheless, additional studies examining the precise mechanism of deguelin modulating the cell cycle are needed.

\section{Deguelin induced tumor cell death by targeting anti- angiogenesis}

To examine the anti-angiogenic efficacy of deguelin in cancer cells, micro-vessel density was measured in xenograft tumors obtained form control or deguelin-treated mice by targeting of the HIF-1 $\alpha$-VEGF signaling pathway. The administration of deguelin evidently decreased tumor vascularization, as reflected by the tumor vessel number/high-power field from 100 to $58 \%$ (31). The chick aortic arch ring assay showed that deguelin at a concentration of $5 \mathrm{nmol}$ significantly reduced the number of endothelial cell sprouts. The chorioallantoic membrane and Matrigel plug assays also confirmed the antiangiogenic activity of deguelin. The treatment of deguelin (1 nmol/egg) suppressed new vessel formation to $46.7 \pm 3.33 \%$ $(n=20)$ in chick embryos. Subsequent experiments were carried out to investigate the inner machinery between deguelin and vessel development in the tumor. It is well-known that VEGF and bFGF are crucial elements in new vessel generation. Western blot analysis suggested that deguelin treatment (100 nmol) arrested HIF-1 $\alpha$ protein synthesis and initiated ubiquitin-mediated protein degradation, resulting in the downregulation of its target, VEGF and bFGF. Further evidence indicated that HIF-1 $\alpha$ degradation was correlated with Hsp90 binding $(32,33)$. The HIF-1 $\alpha$-driven expression of VEGF is significant in vascular endothelial cancer cell growth, while deguelin appears to block tumor angiogenesis by breaking down the HIF-1 $\alpha$-driven, VEGF-induced autocrine/paracrine loops.

Deguelin-induced apoptosis, cell cycle arrest and antiangiogenesis provide novel mechanisms to retinoid research, while preclinical/clinical trials of deguelin are promoted to explore its antitumor efficacy in practice.

A schematic model of deguelin as a novel anti-tumorigenic agent targeting the cell cycle arrest, apoptosis and angiogenic pathways is shown in Fig. 2.

\section{Deguelin was applied in pre-clinical and clinical trials}

In early 1997 , animal experiments confirmed that deguelin decreased tumor incidence from $60 \%$ in the control group to $10 \%$ in the deguelin-treated group at a dose of $33 \mu \mathrm{g} / \mathrm{rat}$, while tumor multiplicity was reduced from 4.2 to 0.1 . At an elevated (330 $\mu \mathrm{g} / \mathrm{rat})$ dose, no tumors were observed in the treatment group (5). Topically-administered deguelin significantly suppressed the multiplicity of skin tumors with 
UVB-induction, indicating its effect as a potential cancer chemopreventive agent (6). In A/J mice, deguelin clearly reduced the tumor multiplicity and volume, as well as the overall tumor burden with exposure to the tobacco-specific carcinogen benzo(a)pyrene (Bap) and other carcinogens, with no detectable toxicity $(7,8)$. Nevertheless, the toxicity of deguelin over a certain dose should not be neglected. Treatment with deguelin, a potential mitochondria complex I inhibitor (34), reduced tyrosine hydroxylase-positive neurons, leading to Parkinson's disease (PD). Kim et al (35) showed that deguelin promoted a PD-like syndrome, mainly by $\mathrm{Src} /$ STAT signaling, since $\alpha$-synuclein (a key protein function in the pathogenesis of PD) was phosphorylated by deguelinactivated Src (35).

Numerous studies conducted on pre-clinical trials have focused on deguelin modification, because of its antitumor activity and some cell toxicity. Through the hydroxylation of $7 \alpha$ carbon or the functional group substitution of 7 carbon of deguelin, several derivatives have been synthesized and their biochemical features, such as solubility and anti-proliferative effects, evaluated. Candidate SH-14 showed the highest apoptotic activity with no detectable effect on PD evoking for clinical use (35). Thus, the modified derivatives successfully reduced the side-effects of deguelin, while maintaining its potential antitumor activity, which is crucial for the systemic clinical evaluation of these novel derivative drugs.

\section{Conclusion}

Generally, the essential changes in tumors are alterations in the genome and protein expression levels that contributed to the biological events involved in tumor malignant phenotype: dysregulated growth stimuli, apoptosis control deletion, cell cycle checkpoint turning off, limitless angiogenesis, immune escape, tissue infiltration and metastasis, as well as insensitivity to chemoradiation.

Deguelin is potentially superior as a natural antitumor agent for various types of cancer cells: Colo 16 and SRB-12 skin cancer cells, normal HBE and BEAS-2B HBE cells, as well as MKN-28 and SNU-484 gastric cancer cells. At times, however, it is highly toxic, being closely correlated with the pathogenesis of Parkinson's disease. However, whether deguelin is an excellent antitumor agent or a poisonous drug depends solely on its dose. For example, deguelin $(4 \mathrm{mg} / \mathrm{kg})$ significantly inhibits the growth of $\mathrm{H} 1299$ xenograft tumors $\left[\left(115.9 \mathrm{~mm}^{3}\right.\right.$ (treatment group) vs. $798 \mathrm{~mm}^{3}$ (control group)] without detectable toxic effects (32), whereas a long-term and high-dose deguelin or rotenone administration resulted in a PD-like syndrome in rats $(34,36)$. Despite these findings, deguelin is beneficial. 17-Allylamino-17-demethoxygeldanamycin (17-AAG), a small-molecular inhibitor of Hsp90 currently in phase II clinical trails, demonstrated various side-effects with clinical symptoms (37,38), whereas deguelin demonstrated antitumor activity at a certain dose with no toxicity to healthy cells. Additionally, new derivatives were found to be promising in overcoming the probable toxicity of deguelin in the future.

As mentioned previously, deguelin has been shown to induce cancer cell death or suppress tumor growth by PI3K-Akt, IKK-IкB $\alpha-N F-\kappa B$, MAPK-mTOR-survivin-mediated apoptosis, p27-cyclinE-pRb-E2F1-mediated cell cycle arrest and HIF1 $\alpha$-VEGF-mediated anti-angiogenesis. Deguelin is essential in such signaling pathways, mainly due to its being a special inhibitor of Hsp90 chaperon function. Its binding to Hsp90 leads to a decreased expression of numerous oncogenic proteins, including MEK1/2, Akt, HIF1 $\alpha, \mathrm{COX}-2$ and NF-kB.

The aim of future studies is to investigate the precise mechanism of deguelin-induced apoptosis and anti-angiogenesis, which are likely to provide a guide for further clinical trials and the development of new derivatives. The clarification of the molecular mechanism of deguelin-induced cell death and the pre-clinical and clinical trials indicate that deguelin has a marked potential for cancer chemoprevention and chemotherapy. Thus, further clinical experiments should be conducted for novel agent development.

\section{Acknowledgements}

This study was supported by the National Natural Science Foundation of China (no. 30873145).

\section{References}

1. Yoshitani SI, Tanaka T, Kohno $\mathrm{H}$ and Takashima S: Chemoprevention of azoxymethane-induced rat colon carcinogenesis by dietary capsaicin and rotenone. Int J Oncol 19: 929-939, 2001.

2. Thamilselvan V, Menon M and Thamilselvan S: Anticancer efficacy of deguelin in human prostate cancer cells targeting glycogen synthase kinase-3 beta/beta-catenin pathway. Int $\mathbf{J}$ Cancer 129: 2916-2927, 2011.

3. Matsuda H, Yoshida K, Miyagawa K, et al: Rotenoids and flavonoids with anti-invasion of HT1080, anti-proliferation of U937, and differentiation-inducing activity in HL-60 from Erycibe expansa. Bioorg Med Chem 15: 1539-1546, 2007.

4. Mulcahy P, O'Doherty A, Paucard A, O'Brien T, Kirik D and Dowd E: Development and characterisation of a novel rat model of Parkinson's disease induced by sequential intranigral administration of AAV- $\alpha$-synuclein and the pesticide, rotenone. Neuroscience 203: 170-179, 2012.

5. Udeani GO, Gerhauser C, Thomas CF, et al: Cancer chemopreventive activity mediated by deguelin, a naturally occurring rotenoid. Cancer Res 57: 3424-3428, 1997.

6. Gills JJ, Kosmeder J II, Moon RC, Lantvit DD and Pezzuto JM: Effect of deguelin on UVB-induced skin carcinogenesis. J Chemother 17: 297-301, 2005.

7. Yan Y, Wang Y, Tan Q, Lubet RA and You M: Efficacy of deguelin and silibinin on benzo(a)pyrene-induced lung tumorigenesis in A/J mice. Neoplasia 7: 1053-1057, 2005.

8. Lee HY, Oh SH, Woo JK, et al: Chemopreventive effects of deguelin, a novel Akt inhibitor, on tobacco-induced lung tumorigenesis. J Natl Cancer Inst 97: 1695-1699, 2005.

9. Long JS and Ryan KM: New frontiers in promoting tumour cell death: targeting apoptosis, necroptosis and autophagy. Oncogene: Feb 6, 2012 (Epub ahead of print).

10. Chun KH, Kosmeder JW II, Sun S, et al: Effects of deguelin on the phosphatidylinositol 3-kinase/Akt pathway and apoptosis in premalignant human bronchial epithelial cells. J Natl Cancer Inst 95: 291-302, 2003.

11. Chen Y, Wu Q, Cui GH, Chen YQ and Li R: Deguelin blocks cells survival signal pathways and induces apoptosis of HL-60 cells in vitro. Int J Hematol 89: 618-623, 2009.

12. Bortul R, Tazzari PL, Billi AM, et al: Deguelin, A PI3K/AKT inhibitor, enhances chemosensitivity of leukaemia cells with an active PI3K/AKT pathway. Br J Haematol 129: 677-686, 2005.

13. Chu ZH, Liang XH, Zhou XL, Huang RF, Zhan Q and Jiang JW: Effects of deguelin on proliferation and apoptosis of MCF-7 breast cancer cells by phosphatidylinositol 3-kinase/Akt signaling pathway. Zhong Xi Yi Jie He Xue Bao 9: 533-538, 2011 (In Chinese).

14. Lee HY: Molecular mechanisms of deguelin-induced apoptosis in transformed human bronchial epithelial cells. Biochem Pharmacol 68: 1119-1124, 2004. 
15. Li R, Chen Y, Shu WX, Chen Z and Ke WJ: Involvement of SRC-3 in deguelin-induced apoptosis in Jurkat cells. Int J Hematol 89: 628-635, 2009.

16. Nair AS, Shishodia S, Ahn KS, Kunnumakkara AB, Sethi G and Aggarwal BB: Deguelin, an Akt inhibitor, suppresses IkappaBalpha kinase activation leading to suppression of NF-kappaB-regulated gene expression, potentiation of apoptosis, and inhibition of cellular invasion. J Immunol 177: 5612-5622, 2006.

17. Dat NT, Lee JH, Lee K, Hong YS, Kim YH and Lee JJ: Phenolic constituents of Amorpha fruticosa that inhibit NF-kappaB activation and related gene expression. J Nat Prod 71: 1696-1700, 2008.

18. Geeraerts B, Vanhoecke B, Vanden Berghe W, Philippe J, Offner F and Deforce D: Deguelin inhibits expression of IkappaBalpha protein and induces apoptosis of B-CLL cells in vitro. Leukemia 21: 1610-1618, 2007.

19. Chen WH, Chen Y and Cui GH: Deguelin inhibits expression of IkappaBalpha protein in Raji and U937 cells. Acta Pharmacol Sin 27: 485-490, 2006.

20. Dell'Eva R, Ambrosini C, Minghelli S, Noonan DM, Albini A and Ferrari N: The Akt inhibitor deguelin, is an angiopreventive agent also acting on the NF-kappaB pathway. Carcinogenesis 28 : 404-413, 2007.

21. Jin Q, Feng L, Behrens C, et al: Implication of AMP-activated protein kinase and Akt-regulated survivin in lung cancer chemopreventive activities of deguelin. Cancer Res 67: 11630-11639, 2007

22. Xiang X, Saha AK, Wen R, Ruderman NB and Luo Z: AMP-activated protein kinase activators can inhibit the growth of prostate cancer cells by multiple mechanisms. Biochem Biophys Res Commun 321: 161-167, 2004.

23. Cao C, Lu S, Kivlin R, et al: AMP-activated protein kinase contributes to UV- and $\mathrm{H}_{2} \mathrm{O}_{2}$-induced apoptosis in human skin keratinocytes. J Biol Chem 283: 28897-28908, 2008.

24. Okoshi R, Ozaki T, Yamamoto $\mathrm{H}$, et al: Activation of AMP-activated protein kinase induces $\mathrm{p} 53$-dependent apoptotic cell death in response to energetic stress. J Biol Chem 283 : 3979-3987, 2008

25. Zheng QY, Jin FS, Yao C, Zhang T, Zhang GH and Ai X: Ursolic acid-induced AMP-activated protein kinase (AMPK) activation contributes to growth inhibition and apoptosis in human bladder cancer T24 cells. Biochem Biophys Res Commun 419: 741-747, 2012.

26. Chen MB, Shen WX, Yang Y, Wu XY, Gu JH and Lu PH: Activation of AMP-activated protein kinase is involved in vincristine-induced cell apoptosis in B16 melanoma cell. J Cell Physiol 226: 1915-1925, 2011.
27. Murillo G, Salti GI, Kosmeder JW II, Pezzuto JM and Mehta RG: Deguelin inhibits the growth of colon cancer cells through the induction of apoptosis and cell cycle arrest. Eur J Cancer 38: 2446-2454, 2002.

28. Lee HY, Suh YA, Kosmeder JW, Pezzuto JM, Hong WK and Kurie JM: Deguelin-induced inhibition of cyclooxygenase-2 expression in human bronchial epithelial cells. Clin Cancer Res 10: 1074-1079, 2004

29. Murillo G, Peng X, Torres KE and Mehta RG: Deguelin inhibits growth of breast cancer cells by modulating the expression of key members of the Wnt signaling pathway. Cancer Prev Res (Phila) 2: 942-950, 2009.

30. Liu HL, Chen Y, Cui GH, Wu QL, Chen WH and He J: Effect of deguelin on proliferation and apoptosis of lymphoma Daudi cells and its mechanism. Zhonghua Zhong Liu Za Zhi 29: 176-180, 2007 (In Chinese).

31. Oh SH, Woo JK, Jin Q, et al: Identification of novel antiangiogenic anticancer activities of deguelin targeting hypoxia-inducible factor-1 alpha. Int J Cancer 122: 5-14, 2008.

32. Oh SH, Woo JK, Yazici YD, et al: Structural basis for depletion of heat shock protein 90 client proteins by deguelin. J Natl Cancer Inst 99: 949-961, 2007.

33. Kim WY, Oh SH, Woo JK, Hong WK and Lee HY: Targeting heat shock protein 90 overrides the resistance of lung cancer cells by blocking radiation-induced stabilization of hypoxia-inducible factor-1alpha. Cancer Res 69: 1624-1632, 2009.

34. Caboni P, Sherer TB, Zhang N, et al: Rotenone, deguelin, their metabolites, and the rat model of Parkinson's disease. Chem Res Toxicol 17: 1540-1548, 2004

35. Kim WY, Chang DJ, Hennessy B, et al: A novel derivative of the natural agent deguelin for cancer chemoprevention and therapy. Cancer Prev Res (Phila) 1: 577-587, 2008.

36. Greenamyre JT, Cannon JR, Drolet R and Mastroberardino PG: Lessons from the rotenone model of Parkinson's disease. Trends Pharmacol Sci 31: 141-143, 2010.

37. Banerji U, O'Donnell A, Scurr M, et al: Phase I pharmacokinetic and pharmacodynamic study of 17-allylamino, 17-demethoxygeldanamycin in patients with advanced malignancies. J Clin Oncol 23: 4152-4161, 2005

38. Grem JL, Morrison G, Guo XD, et al: Phase I and pharmacologic study of 17-(allylamino)-17-demethoxygeldanamycin in adult patients with solid tumors. J Clin Oncol 23: 1885-1893, 2005. 